\title{
Contrast-Associated Acute Kidney Injury: Advances and Challenges
}

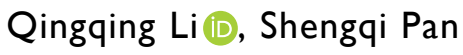 \\ Department of Intensive Care Medicine, Qingdao Municipal Hospital, Qingdao, People’s Republic of China
}

Correspondence: Qingqing Li, Department of Intensive Care Medicine, Qingdao Municipal Hospital, Qingdao, People's Republic of China, Email2276105001@qq.com

\begin{abstract}
A decrease in renal function that follows intravascular administration of contrast medium (CM) within a few days is reported as contrast-induced acute kidney injury (CI-AKI). In clinical practice, the imaging procedure is frequently deferred when clinicians consider that the renal risks caused by $\mathrm{CM}$ outweigh the benefits of enhanced imaging. However, with an in-depth understanding of AKI and contrast medium, scholars have realized that the decrease in renal function after CM is caused by contrast medium factors and noncontrast medium factors (such as anemia and hemodynamic instability). Therefore, acute kidney injury caused by CM has been overestimated in the past. The term "contrast-associated acute kidney injury (CA-AKI)" has been increasingly used to indicate AKI after intravascular administration of contrast medium compared with CI-AKI. CA-AKI can increase the risk of death and chronic kidney disease. However, its pathophysiological mechanism has not been fully elucidated, and the effectiveness of various preventive and therapeutic measures have been questioned. These present challenges for us. In this article, we will review the diagnostic criteria, epidemiology, risk factors, pathophysiological mechanisms and treatment of CA-AKI to provide optimized imaging procedures in clinical practice.
\end{abstract}

Keywords: contrast-associated acute kidney injury, contrast medium, acute kidney injury

Contrast medium was first reported to cause acute kidney injury in $1954,{ }^{1}$ and the disease is now well known by clinicians. Before undergoing intravascular administration of contrast medium, the patient's renal function is assessed. Acute kidney injury is a seriously illness that can increase the mortality of patients and the incidence of chronic renal insufficiency. Data from the University of Pittsburgh Medical Center showed that the 30-d in-hospital mortality increased, even if creatinine was slightly elevated. ${ }^{2}$ In addition to impairing the health of patients, CA-AKI will also increase the economic burden on families and society. ${ }^{3}$ Therefore, it is vital to understand the advances in CA-AKI to strengthen its prevention and treatment.

\section{Diagnostic Criteria}

Contrast-induced nephropathy (CIN) was previously characterized by an absolute increase in plasma creatinine of $0.5 \mathrm{mg} / \mathrm{dl}(44 \mu \mathrm{mol} / \mathrm{L})$ or $\geq 25 \%$ of the baseline creatinine value within 2 to 5 days after the application of intravascular contrast medium (CM). ${ }^{4,5}$ In 2012, the Kidney Disease Improving Global Outcomes (KDIGO) proposed the term "contrast-induced acute kidney injury (CI-AKI)" and defined it as an increase in serum creatinine value to more than 1.5 times the baseline creatinine value within 7 days after contrast exposure, or an increase of $\geq 0.3 \mathrm{mg} / \mathrm{dl}$ $(26.5 \mu \mathrm{mol} / \mathrm{L})$ in creatinine within $48 \mathrm{~h}$, or a decrease to $0.5 \mathrm{~mL} / \mathrm{kg} / \mathrm{h}$ for 6 hours in the urine volume after the application of $\mathrm{CM}^{6}$

In recent years, $\mathrm{AKI}$ has been shown to be induced by factors other than $\mathrm{CM}$ after applying intravascular $\mathrm{CM}$, such as hypotension, low cardiac output, sepsis, anemia and so forth. Therefore, the term "contrast-associated acute kidney injury" has gained favor. ${ }^{7}$ 


\section{Epidemiology}

Numerous studies have focused on AKI incidence after exposure to $\mathrm{CM}$, with the overall findings showing significant differences, ranging from $1.3 \%$ to $33.3 \%{ }^{8}$ Such differences in various studies may be caused by the inclusion or exclusion of AKI induced by non-CM factors as well as the different AKI definitions and study populations. Because non-CM factors also affect renal function, the correlation between $\mathrm{CM}$ and $\mathrm{AKI}$ and its severity has been questioned recently.

First, a series of retrospective studies containing control groups (patients without CM exposure) were conducted to determine whether CM could cause AKI. A meta-analysis of 13 retrospective controlled studies, with 25,950 individuals, showed that intravenous CM had no impact on the incidence of AKI, with similar mortality and dialysis observed. ${ }^{4}$ However, the primary health conditions of patients in the trial and control groups were different, and it might be misleading to directly compare the incidence of AKI between the two groups.

To minimize the baseline level of patients from the trial and control groups, paired retrospective studies were conducted based on propensity scores. Davenport et $\mathrm{al}^{9}$ matched 19 covariates among 17,652 individuals and found that hypotonic CM had a significant impact on the occurrence of AKI after CT $(P=0.04)$, and the risk of AKI after angiography was increased with a decrease in eGFR. Wilhelm-Leen et $\mathrm{al}^{10}$ conducted a large-scale cohort study on inpatients based on whether $\mathrm{CM}$ was applied. After calibration, the results showed that the incidence of AKI in patients with $\mathrm{CM}$ was $5.5 \%$ while that in patients without $\mathrm{CM}$ was $5.6 \%$, with an odds ratio (OR) of 0.93 and a $95 \%$ confidence interval $(95 \% \mathrm{CI})$ of $0.88-0.97$, which indicated that $\mathrm{CM}$ did not promote the occurrence of AKI. Hinson et $\mathrm{al}^{11}$ conducted a study among 17,934 patients and found that $\mathrm{CM}$ was not associated with the increased incidence of AKI, which was similar in all subgroup analyses, regardless of baseline renal function, direct comparison or comparison after propensity score matching. Currently, there are three kinds of common propensity scores, but many underlying factors can affect renal function, and no one propensity score can fully cover all relevant aspects. In addition, the above studies were retrospective, individuals with renal insufficiency were unlikely to receive CM clinically, and hydration therapy was performed after CM exposure. So there may be certain limitations in the above results.

Therefore, it is necessary to conduct large-scale prospective randomized controlled studies, but ethics issues would be a major challenge. Currently, it can be confirmed that the risk of CI-AKI has been overestimated. Recently, a paper published in Chest also supports this conclusion. ${ }^{12}$

The existing evidence suggests that the risk of CI-AKI after intravenous CM exposure can be negligible in adult patients with an estimated glomerular filtration rate (eGFR) $\geq 60 \mathrm{~mL} / \mathrm{min} / 1.73 \mathrm{~m}^{2}$ or minor renal dysfunction (eGFR: $45-59 \mathrm{~mL} / \mathrm{min} /$ $\left.1.73 \mathrm{~m}^{2}\right) .{ }^{13}$ Recently, the results of a study focusing on children demonstrated that the risk of contrast-induced nephropathy was not increased after applying CM with normal renal function or minor renal dysfunction (eGFR $\geq 60 \mathrm{~mL} / \mathrm{min} / 1.73 \mathrm{~m}^{2}$ ), confirming that $\mathrm{CM}$ has little impact on patients with normal renal function. Although there are differences in the results of propensity score studies, it is inevitable that patients with eGFR $<30 \mathrm{~mL} / \mathrm{min} / 1.73 \mathrm{~m}^{2}$ have the highest risk of CI-AKI. ${ }^{15}$ A consensus has revealed that intravenous CM exposure cannot increase the risk of CI-AKI in patients with an eGFR of 30$44 \mathrm{~mL} / \mathrm{min} / 1.73 \mathrm{~m}^{2},{ }^{15,16}$ but we need further research on diabetic patients.

\section{Risk Factors}

\section{Basic Health Conditions of Patients}

A decreased estimated glomerular filtration rate (eGFR) is an independent risk factor for CA-AKI. In addition, Rudnick et $\mathrm{al}^{17}$ found that diabetic patients were prone to CA-AKI more than 20 years ago. However, diabetes is not an independent risk factor for CA-AKI, which may be caused by diabetic nephropathy. In addition to the above two traditional risk factors, advanced age, hypertension, congestive heart failure (HF) and anemia can also increase the incidence of CA-AKI and the short-term and long-term complications and mortality of patients. ${ }^{16,18,19}$ High-density lipoprotein (HDL) possesses anti-inflammatory and antioxidant effects, further promoting the function and repair of endothelial cells (ECs). It has been found that the level of HDL is negatively correlated with the risk of CA-AKI after coronary angiography. ${ }^{20,21}$ However, the specific biological basis is unclear thus far, which may be related to antioxidation and renal vessel dilation. 


\section{Medical Measures}

The contrast medium is divided into three types according to its osmolality, namely, high-osmolar, iso-osmolar and lowosmolar contrast medium. High-osmolar contrast agents have been found to significantly increase the incidence of CA-AKI; hence, this kind of $\mathrm{CM}$ is rarely adopted at present. Compared with the hypotonic contrast agent, Aspelin et $\mathrm{al}^{22}$ found that the risk of CA-AKI induced by isotonic contrast agent was lower. However, similar conclusions are not observed in other studies. For instance, Rudnick et al ${ }^{23,24}$ found no difference in AKI of patients with renal insufficiency after applying isotonic and hypotonic contrast medium. Han et $\mathrm{al}^{25}$ obtained the same results in a meta-analysis, even for diabetic patients. In addition, the dose of CM is also related to CA-AKI, implying that a higher CM dose corresponds to a higher risk of CAAKI. ${ }^{22}$ Studies have shown that a CM dose less than three times the creatinine clearance rate (Ccr) could reduce the incidence of CA-AKI. ${ }^{26,27}$ Moreover, repeated administration of CM within 72 hours would increase the risk of CA-AKI. ${ }^{28}$ In arterial CM administration, it is significant to discriminate the second exposure (where the CM reaches the kidney in a diluted form) from the first exposure (where the kidney is exposed to relatively undiluted $\mathrm{CM}$ ). The second exposure occurs when $\mathrm{CM}$ is injected into the cor dextrum and pulmonary artery and selectively perfused into the suprarenal aortic branches (subclavian, brachial and mesenteric arteries). The first exposure occurs when the $\mathrm{CM}$ reaches the cor sinistrum and suprarenal aorta and selectively arrives in the renal artery. Generally, the risk of AKI after intravenous use of contrast medium is lower than that of arterial contrast medium. Because patients receiving coronary angiography have relatively serious pathogenetic conditions, they are high-risk groups for CA-AKI. Furthermore, CM is commonly infused through arteries, and the first and second renal exposures occur simultaneously during coronary angiography. Therefore, the risk of CA-AKI in patients after coronary angiography is far higher than that after enhanced $\mathrm{CT}^{29}{ }^{29}$ is worth noting that nephrotoxic drugs often have to be applied to treat diseases in the clinic, thereby increasing the occurrence of CA-AKI. For instance, nonsteroidal anti-inflammatory drugs can constrict renal blood vessels and cause renal interstitial fibrosis; aminoglycosides have direct toxic effects on kidney tubules. Recently, Ma et $\mathrm{al}^{19}$ found that renin-angiotensin-aldosterone system inhibitors, which are commonly used to prevent renal complications in patients with hypertension and diabetes, increased the risk of AKI in diabetic patients after coronary angiography. The possible reason is that these drugs can dilate the efferent arteriole of the glomerulus, and the decrease in GFR slows down the clearance of CM and then causes persistent damage to kidneys. Moreover, angiotensin II can induce the production of transforming growth factor $\beta 1$ (TGF- $\beta 1$ ), which could protect renal proximal tubular cells from necrosis, but renin-angiotensin-aldosterone system inhibitors counteract the potential protective effect of TGF- $\beta 1$ in the kidney by inhibiting the production of angiotensin II. Risk factors are shown in Table 1.

Table I High-Risk Factors for CA-AKI

\begin{tabular}{|l|}
\hline Risk Factors for CA-AKI \\
\hline Patient-associated factors \\
\hline - Pre-existing renal function insufficiency \\
- Diabetic nephropathy \\
- Advanced age (> 70 years old) \\
- Hypertension \\
- Cognitive heart failure \\
- Anemia \\
- Periprocedural hypoperfusion \\
\hline Medical procedure-associated factors \\
\hline - High osmolality of iodine agent \\
- Excessive use of iodine agent \\
- Repeated exposure of contrast agent within $72 \mathrm{~h}$ \\
- Imaging techniques \\
- Nephrotoxic drug (NSAIDs, aminoglycosides, ACEl, etc.) \\
\hline
\end{tabular}




\section{Pathophysiological Mechanism}

Although the pathophysiological mechanism of CI-AKI has not been fully clarified, the possible mechanisms are direct toxic effects of CM, renal hemodynamic changes, oxygen-free radicals and inflammatory reactions (Figure 1).

\section{Direct Toxic Effects of CM on Kidneys}

$\mathrm{CM}$ has direct toxic effects on renal tubular epithelial cells and can induce apoptosis and necrosis, which is due to the physicochemical properties of CM. The early injury of renal tubular epithelial cells is characterized by the loss of cell polarity due to the redistribution of the $\mathrm{Na}^{+}-\mathrm{K}^{+}$pump. Pumps on the basolateral side of tubular epithelial cells decrease and those on the luminal surface increase, accompanied by the increasing transport of sodium ions to distal convoluted tubules, thus leading to the contraction of renal blood vessels through tubuloglomerular feedback (Figure 2). With the injury of epithelial cells and shedding from the basement membrane, obstruction would occur in the renal tubules, thus causing a pressure increase within the renal tubules and a decrease in GFR. ${ }^{2,7}$

\section{Effects of CM on Renal Hemodynamics}

$\mathrm{CM}$ can increase vasoconstricting substances (such as endothelin) and decrease vasodilating substances (such as NO and $\mathrm{PGI}_{2}$ ) in plasma, leading to a lessening in global or local blood perfusion of kidneys. ${ }^{2,7}$ Although renal medulla blood flow only accounts for $10 \%$ of renal blood flow, epithelial cells in the thick ascending limb of Henle's loop are in a state of high metabolism. Therefore, ischemia and hypoxia are prone to occur in the outer medulla when renal blood flow decreases. ${ }^{30}$ It is worth noting that the renal parenchyma of patients with CKD and diabetic nephropathy is decreased and the conventional dose of CM can aggravate ischemia of the outer medulla. Moreover, diabetic nephropathy is characterized by renal vascular dysfunction, which increases the sensitivity to vasoconstriction caused by CM. Meanwhile, CM

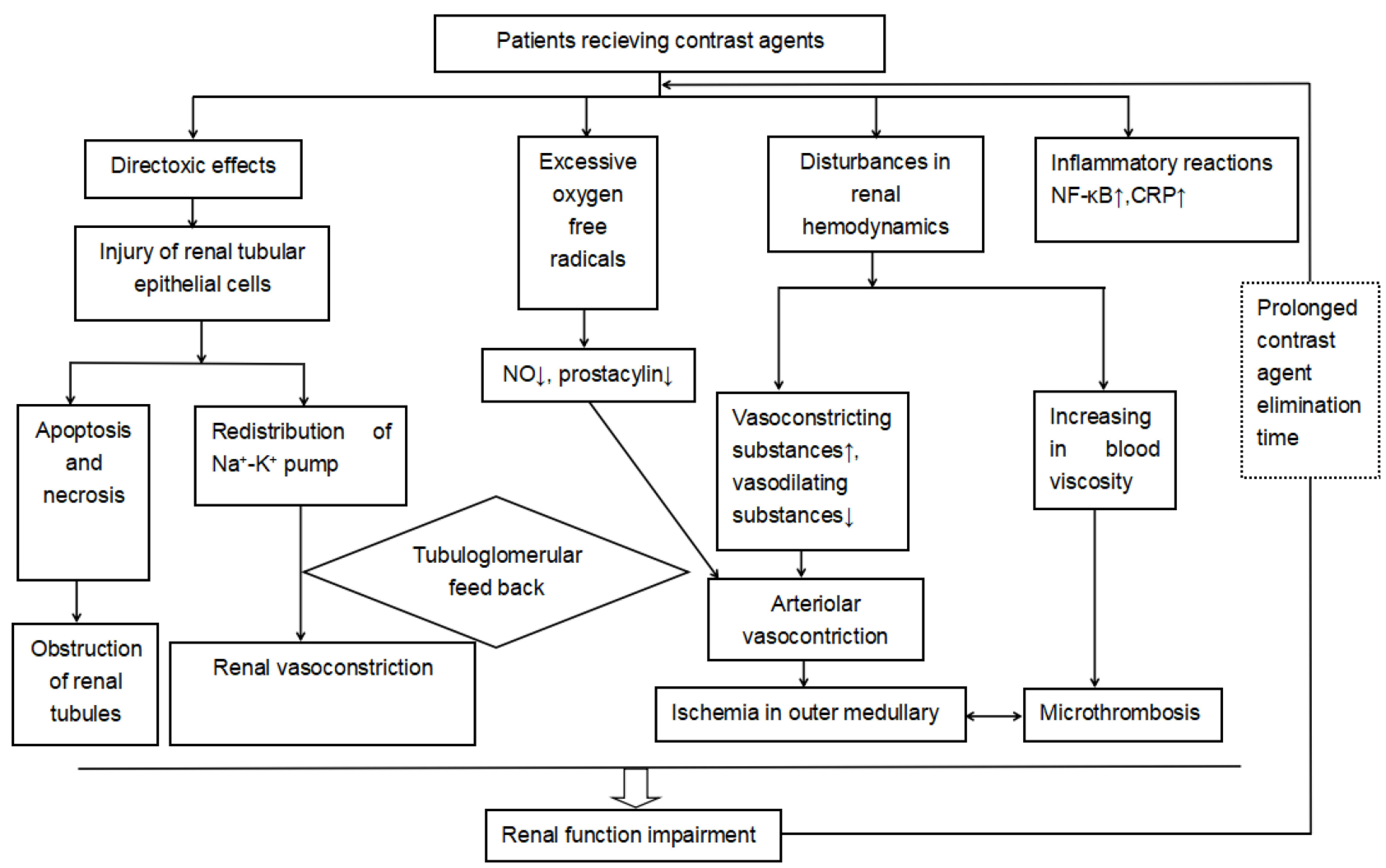

Figure I Mechanisms of Contrast-Associated Acute Kidney Injury. Abbreviations: NO, nitric oxide; CRP, C-reactive protein. 


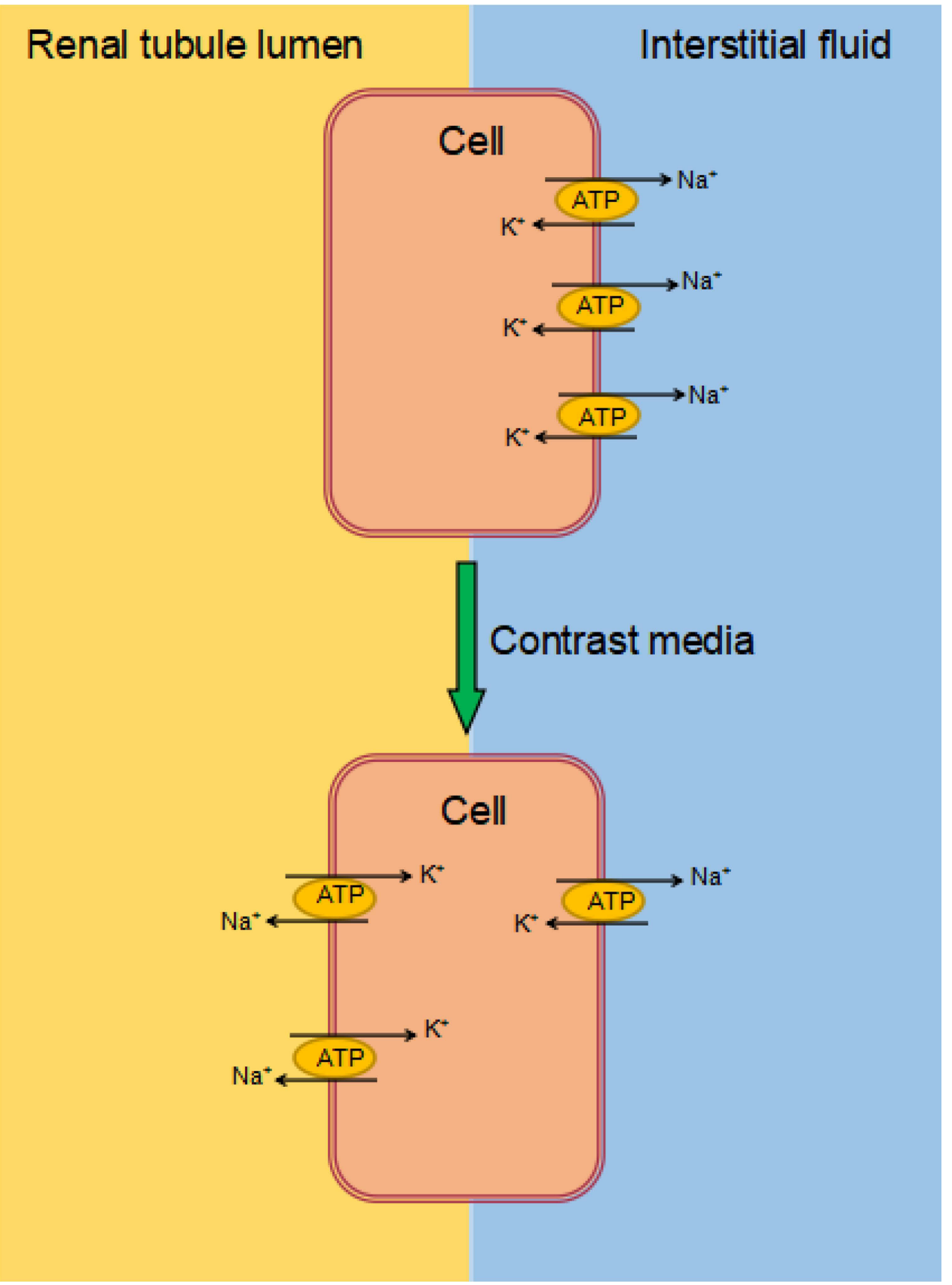

Figure 2 Redistribution of the $\mathrm{Na}^{+}-\mathrm{K}^{+}$pump.

Notes: $\mathrm{CM}$ has direct toxic effects on renal tubular epithelial cells, resulting in the redistribution of the $\mathrm{Na}^{+}-\mathrm{K}^{+}$pump. The pumps deliver from the basolateral to the luminal side of the tubular cells, causing an $\mathrm{Na}^{+}$increase in the tubule lumen.

can increase blood viscosity, ${ }^{31}$ slow microcirculatory blood flow, and affect blood osmotic pressure, thus reducing red blood cell plasticity and increasing the risk of microthrombosis.

\section{Increases the Production of Oxygen Free Radicals}

$\mathrm{CM}$ has been reported to increase the production of oxygen free radicals by reducing the biological activity of renal tubular epithelial cells and promoting the peroxidation of lipids. Moreover, it also reduces the activities of antioxidant enzymes and superoxide dismutase, which lead to an increase in the production of reactive oxygen species (ROS). ${ }^{32}$ Excessive ROS could cause damage to renal tubular epithelial cells and adjacent cells. In addition, with increases in 
ROS, nitric oxide (NO) and prostaglandins decrease because the activities of nitric oxide synthase (NOS) and prostacyclin synthase are hindered by ROS then renal blood vessels contract, ${ }^{33}$ thus aggravating cell hypoxia and renal insufficiency. ${ }^{34}$

\section{Inflammatory Reactions}

$\mathrm{NF}-\kappa \mathrm{B}$ is a transcription factor involved in immunity, stress response, apoptosis and differentiation. In recent years, NF$\kappa \mathrm{B}$ has been shown to play a vital role in the occurrence and development of $\mathrm{AKI} .{ }^{35}$ Machado et $\mathrm{al}^{36}$ detected a large amount of NF- $\mathrm{KB}$ expression in a rat contrast medium model, and their results showed that reducing the activity of NF$\kappa \mathrm{B}$ can alleviate the inflammatory response and oxidative stress injury in AKI. The results of a prospective cohort study including 423 patients suggested that patients in the high C-reactive protein (CRP) group had a higher relative risk of CIN. ${ }^{37}$ These data indicate that inflammatory reactions may play an important role in the pathogenesis of CA-AKI.

\section{Prophylaxis and Treatment of CA-AKI}

Hydration is the basic prophylaxis method for CA-AKI. According to the 2015 Guidelines on Acute Coronary Syndrome and 2018 European Society of Cardiology Guidelines on Myocardial Revascularization, intravenous hydration treatment is recommended for CA-AKI patients with an estimated glomerular filtration rate (eGFR) $<60 \mathrm{~mL} / \mathrm{min} / 1.73 \mathrm{~m}^{2}$ under the condition of $12 \mathrm{~h}$ before coronary angiography and $24 \mathrm{~h}$ after coronary angiography. ${ }^{38,39} \mathrm{In}$ recent studies, hydration therapy has also been shown to reduce the incidence of CA-AKI in patients with eGFR $\geq 60 \mathrm{~mL} / \mathrm{min} / 1.73 \mathrm{~m}^{2} .{ }^{40}$ However, excessive transfusion may increase the risk of heart failure, arrhythmia and short-term death in high-risk patients. ${ }^{41}$ Therefore, the implementation of hydration therapy should be guided according to the central venous pressure (CVP), left ventricular end diastolic pressure (LVEDP), bioimpedance and urine volume. A prospective randomized double-blind trial found that CVP-guided hydration therapy outperforms the standard hydration scheme for patients with coronary angiography. ${ }^{42}$ In addition, similar guiding effects of LVEDP-guided hydration were observed in the POSEIDON trial. ${ }^{5}$ The evaluation of the bioimpedance vector level at admission contributes to adjustments of vascular content, and the incidence of CA-AKI decreases after angiographic surgery. ${ }^{43}$ The RenalGuard system is a device that optimizes intravenous hydration treatment by matching the fluid infusion volume with the patient's urination volume, which can enable the rapid discharge of CM by maintaining a high urination volume. Briguori et al ${ }^{44}$ found that compared with LVEDP-guided hydration therapy, RenalGuard-guided hydration therapy led to a decreased incidence of CA-AKI and pulmonary edema (RR: $0.56,95 \%$ CI: $0.39-0.79, P=0.036$ ). However, the results of a prospective randomized controlled noninferiority trial concluded that 8 out of 307 patients (2.6\%) who did not receive hydration therapy developed AKI and 8 out of 296 patients $(2.7 \%)$ who received hydration therapy developed CA-AKI; therefore, hydration therapy did not reduce the incidence of CA-AKI $(P=0.4710) .{ }^{45}$ Although this was a single-center study, it included a population from all over the Netherlands; thus, the results are worthy of wide attention. Moreover, controversies remain regarding the type of liquid used in hydration therapy. Currently, the oral administration of liquid alone is not recommended, and intravenous rehydration is often required. For intravenous rehydration, $1.4 \%$ sodium bicarbonate and $0.9 \%$ normal saline are commonly administered fluids. Previous studies with a small sample size have proposed that the intravenous application of sodium bicarbonate is better than saline in reducing the incidence of CA-AKI. ${ }^{46,47}$ However, Weisbord et al ${ }^{48}$ recently conducted a multicenter study that included 5177 individuals in which the subjects were infused separately with $1.26 \%$ sodium bicarbonate and $0.9 \%$ saline, and the results showed no difference in the incidence of CA-AKI between the two groups and similar mortality and incidence of renal replacement therapy.

$\mathrm{N}$-acetylcysteine (NAC) is a free radical scavenger containing a sulfhydryl group that can promote glutathione synthesis and regulate cell metabolism; thus, it possesses antioxidative stress and antitoxicity effects. In addition, NAC can also promote the release of $\mathrm{NO}$ and reduce the production of angiotensin by inhibiting the activity of angiotensinconverting enzymes (ACEs). Then, these substances act on the renal cortex and medulla microcirculation directly or indirectly and ultimately attenuate the renal vasoconstriction caused by $\mathrm{CM}^{49}{ }^{4}$ Therefore, whether NAC can prevent the occurrence of CA-AKI has been a research hotspot. Xie et $\mathrm{al}^{50}$ found that acetylcysteine could significantly reduce the incidence of AKI after angiography (OR: 0.78, 95\% CI: 0.68-0.90, $\left.I^{2}=37.3 \%\right)$. However, a prospective study showed that the incidence of CIN did not differ between the NAC $(1200 \mathrm{mg})$ group and the placebo group $(P=0.81) .{ }^{51}$ Recently, 
a large-scale randomized controlled study published in Lancet revealed that the application of $\mathrm{N}$-acetylcysteine after endovascular angiography did not reduce the mortality and incidence of CA-AKI compared with the placebo group. ${ }^{52}$ The different results of these studies may be related to the different CA-AKI definitions, various basic health conditions and baseline creatinine values of the study population. Other antioxidants, such as sildenafil and tadalafil, ${ }^{52}$ recombinant klotho ${ }^{53}$ and febuxostat, ${ }^{54}$ have been shown to reduce the risk of contrast medium-induced nephropathy in animal experiments. It is necessary to conduct further clinical studies into the protective effect of these drugs on kidneys in the future.

Statins also have anti-inflammatory effects by interfering with the production of active oxygen or scavenging free radicals. Meanwhile, they can also activate endothelial progenitor cells to protect blood vessels. ${ }^{55}$ High-dose rosuvastatin and atorvastatin can reduce the incidence of CA-AKI. ${ }^{56,57}$ Since 2014, the European Society of Cardiology Guidelines on Myocardial Revascularization has suggested that short-term and high-intensity treatment with statins should be applied to patients with moderate to severe CKD in an attempt to reduce the risk of CI-AKI. Contrary to these findings, the decisive benefits of statins have not been proven in some studies. ${ }^{58,59}$ Due to a lack of numerous control data and the heterogeneity of study populations, contradictory results on the effects of statins on CA-AKI have been reported, and there is currently no consensus on the appropriate time or type of statin treatment.

Furthermore, vasodilators, such as adenosine receptor antagonists, asymmetric dimethylarginine and amlodipine, have benefits in reducing the occurrence of CA-AKI, which is perhaps because they can alleviate CM-induced renal medulla ischemia by mitigating renal vasoconstriction. ${ }^{60}$ In addition, the natural active ingredients in traditional Chinese medicines have presented favorable effects on the prevention and treatment of CA-AKI in laboratory studies. ${ }^{61}$ However, it is necessary to perform more clinical trials for verification. Because iodinated CM is water soluble and distributed outside cells, renal replacement therapy can accelerate the clearance of CM and theoretically reduce the toxic effect of CM. However, a meta-analysis that included 1010 individuals reported that renal replacement therapy has no advantage in reducing CA-AKI, ${ }^{62}$ which may be caused by complications during renal replacement therapy (such as hypotension and inflammatory reaction). Currently, the prophylactic application of renal replacement therapy is not recommended unless fluid overload occurs. ${ }^{63}$

\section{Conclusion}

Acute kidney injury caused by contrast medium has been overestimated, and its true incidence and severity are unknown. At present, various measures for the treatment of acute kidney injury after contrast medium administration remain controversial. CI-AKI is currently diagnosed by creatinine, which has low specificity. Most previous studies have mistaken CA-AKI for CI-AKI, even when data on both CA-AKI patients and CA-AKI patients were included in the same study. We believe that the current diagnosis of CI-AKI accounts for most of the controversy and has limited meaningful progress. Scholars must accurately identify CI-AKI patients and come to a consensus on the definition of CIAKI as soon as possible. Meanwhile, the early identification of high-risk groups, selection of appropriate contrast agents and doses, avoidance or reduction in the use of nephrotoxic drugs, strengthening of follow-up among high-risk groups, and early identification of AKI are essential. Finally, it is necessary to further explore the pathophysiological mechanisms of acute kidney injury caused by contrast medium and discover new measures to prevent and treat AKI based on this. These objectives represent challenges and opportunities.

\section{Disclosure}

The authors report no conflicts of interest in this work.

\section{References}

1. Bartels ED, Brun GC, Gammeltoft A, et al. Acute a nuria following in travenous pyelography in a patient with myelomatosis. Acta Med Scand. 1954;150(4):297-302. doi:10.1111/j.0954-6820.1954.tb18632.x

2. Weisbord SD, Chen H, Stone RA, et al. Associations of increases in serum creatinine with mortality and length of hospital stay after coronary angiography. J AmSoc Nephrol. 2006;17(10):2871-2877. doi:10.1681/ASN.2006030301

3. Pistolesi V, Regolisti G, Morabito S, et al. Contrast medium induced acute kidney injury: a narrative review. J Nephrol. 2018;31(6):797-812. doi:10.1007/s40620-018-0498-y 
4. Mc Donald JS, Mc Donald RJ, Comin J, et al. Frequency of acute kidney injury following intravenous contrast medium administration:a systematic review and meta-analysis. Radiology. 2013;267(1):119-128. doi:10.1148/radiol.12121460

5. Brar SS, Aharonian V, Mansukhani P, et al. Haemo dynamic-guided fluid administration for the prevention of contrast-induced acute kidney injury: the POSEIDON randomised controlled trial. Lancet. 2014;383(9931):1814-1823. doi:10.1016/S0140-6736(14)60689-9

6. Kidney Disease Improving Global Out-comes(KDIGO). Clinical practice guide-line for acute kidney injury. Kidney Int Suppl. $2012 ; 2: 1-138$.

7. Mehran R, Dangas GD, Weisbord SD. Contrast-associated acute kidney injury. N Engl J Med. 2019;380(22):2146-2155. doi:10.1056/ NEJMra1805256

8. Chalikias G, Drosos I, Tziakas DN. Contrast-induced acute kidney injury: an update. Cardiovasc Drugs Ther. 2016;30(2):215-228. doi:10.1007/ s10557-015-6635-0

9. Davenport MS, Khalatbari S, Cohan RH, et al. Contrast material-induced nephrotoxicity and intravenous low-osmolality iodinated contrast material: risk stratification by using estimated glomerular filtration rate. Radiology. 2013;268(3):719-728. doi:10.1148/radiol.13122276

10. Wilhelm-Leen E, Montez-Rath ME, Chertow G. Estimating the risk of radiocontrast-associated nephropathy. J Am Soc Nephrol. 2017;28 (2):653-659. doi:10.1681/ASN.2016010021

11. Hinson JS, Ehmann MR, Fine DM, et al. Risk of acute kidney injury after intravenous contrast media administration. Ann Emerg Med. 2017;69 (5):577-586.e4. doi:10.1016/j.annemergmed.2016.11.021

12. Lakhal K, Robert-Edan V, Ehrmann S. In the name of contrast-induced acute kidney injury. Chest. 2020;157(4):751-752. doi:10.1016/j. chest.2019.12.009

13. Rudnick MR, Leonberg-Yoo AK, Litt HI, et al. The controversy of contrast-induced nephropathy with intravenous contrast: what is the risk? Am J Kidney Dis. 2020;75(1):105-113. doi:10.1053/j.ajkd.2019.05.022

14. Gilligan LA, Davenport MS, Trout AT, et al. Risk of acute kidney injury following contrast-enhanced ct in hospitalized pediatric patients: a propensity score analysis. Radiology. 2020;294(3):548-556. doi:10.1148/radiol.2020191931

15. Allen BC, Oto A, Akin O, et al. Post-treatment surveillance of bladder cancer. J Am Coll Radiol. 2019;16(11S):S417-S427. doi:10.1016/j. jacr.2019.05.026

16. van der Molen AJ, Reimer P, Dekkers IA, et al. Post-contrast acute kidney injury. Part 2: risk stratification, role of hydration and other prophylactic measures, patients taking metformin and chronic dialysis patients: recommendations for updated ESUR Contrast Medium Safety Committee guidelines. Eur Radiol. 2018;28(7):2856-2869. doi:10.1007/s00330-017-5247-4

17. Rudnick MR, Goldfarb S, Wexler L, et al. Nephrotoxicity of ionic and nonionic contrast media in 1196 patients: a randomized trial. The iohexol cooperative study. Kidney Int. 1995;47(1):254-261. doi:10.1038/ki.1995.32

18. Rudnick M, Feldman H. Contrast-induced nephropathy: what are the true clinical consequences? Clin J Am Soc Nephrol. 2008;3(1):263-272. doi:10.2215/CJN.03690907

19. Ma M, Wan X, Gao M, et al. Renin-angiotensin-aldosterone system blockade is associated with higher risk of contrast-induced acute kidney injury in patients with diabetes. Aging. 2020;12(7):5858-5877. doi:10.18632/aging.102982

20. Smith LE, Smith DK, Blume JD, et al. High-density lipoprotein cholesterol concentration and acute kidney injury after cardiac surgery. J Am Heart Assoc. 2017;6(12):e006975. doi:10.1161/JAHA.117.006975

21. Park HS, Kim CJ, Hwang BH, et al. HDL cholesterol level is associated with contrast induced acute kidney injury in chronic kidney disease patients undergoing PCI. Sci Rep. 2016;6:35774. doi:10.1038/srep35774

22. Aspelin P, Aubry P, Fransson SG, et al. Nephrotoxic effects in high-risk patients undergoing angiography. $N$ Engl $J$ Med. $2003 ; 348(6): 491-499$. doi:10.1056/NEJMoa021833

23. Rudnick MR, Davidson C, Laskey W, et al. Nephrotoxicity of iodixanol versus ioversol in patients with chronic kidney disease: the Visipaque Angiography/Interventions with Laboratory Outcomes in Renal Insufficiency (VALOR) trial. Am Heart J. 2008;156(4):776-782. doi:10.1016/j. ahj.2008.05.023

24. Solomon RJ, Natarajan MK, Doucet S, et al. Cardiac Angiography in Renally Impaired Patients (CARE) study: a randomized double-blind trial of contrast-induced nephropathy in patients with chronic kidney disease. Circulation. 2007;115(25):3189-3196. doi:10.1161/ CIRCULATIONAHA.106.671644

25. Han XF, Zhang XX, Liu KM, et al. Contrast-induced nephropathy in patients with diabetes mellitus between iso- and low-osmolar contrast media: a meta-analysis of full-text prospective, randomized controlled trials. PLoS One. 2018;13(3):e0194330. doi:10.1371/journal.pone.0194330

26. Gurm HS, Dixon SR, Smith DE, et al. Renal function-based contrast dosing to define safe limits of radiographic contrast media in patients undergoing percutaneous coronary interventions. J Am Coll Cardiol. 2011;58(9):907-914. doi:10.1016/j.jacc.2011.05.023

27. Kooiman J, Seth M, Share D, et al. The association between contrast dose and renal complications post PCI across the continuum of procedural estimated risk. PLoS One. 2014;9(3):e90233. doi:10.1371/journal.pone.0090233

28. Maioli M, Toso A, Gallopin M, et al. Preprocedural score for risk of contrast-induced nephropathy in elective coronary angiography and intervention. J Cardiovasc Med. 2010;11(6):444-449. doi:10.2459/JCM.0b013e328335227c

29. Sgura FA, Bertelli L, Monopoli D, et al. Mehran contrast-induced nephropathy risk score predicts short- and long-term clinical outcomes in patients with ST-elevation-myocardial infarction. Circ Cardiovasc Interv. 2010;3(5):491-498. doi:10.1161/CIRCINTERVENTIONS.110.955310

30. Heyman SN, Rosen S, Rosenberger C. Renal parenchymal hypoxia, hypoxia adaptation, and the pathogenesis of radiocontrast nephropathy. Clin J Am Soc Nephrol. 2008;3(1):288-296. doi:10.2215/CJN.02600607

31. Wong PC, Li Z, Guo J, Zhang A. Pathophysiology of contrast-induced nephropathy. Int J Cardiol. 2012;158(2):186-192. doi:10.1016/j. ijcard.2011.06.115

32. Nikolsky E, Aymong ED, Dangas G, et al. Radiocontrast nephropathy: identifying the high-risk patient and the implications of exacerbating renal function. Rev Cardiovasc Med. 2003;4(Suppl 1):S7-S14

33. Mamoulakis C, Tsarouhas K, Fragkiadoulaki I, et al. Contrast-induced nephropathy: basic concepts, pathophysiological implications and prevention strategies. Pharmacol Ther. 2017;180:99-112. doi:10.1016/j.pharmthera.2017.06.009

34. Heyman SN, Rosen S, Khamaisi M, et al. Reactive oxygen species and the pathogenesis of radiocontrast-induced nephropathy. Invest Radiol. 2010;45(4):188-195. doi:10.1097/RLI.0b013e3181d2eed8 
35. Zhang D, Li Y, Liu Y, et al. Paclitaxel ameliorates lipopolysaccharide-induced kidney injury by binding myeloid differentiation protein-2 to block Toll-like receptor 4-mediated nuclear factor- $\kappa \mathrm{B}$ activation and cytokine production. J Pharmacol Exp Ther. 2013;345(1):69-75. doi:10.1124/ jpet.112.202481

36. Machado RA, Constantino Lde S, Tomasi CD, et al. Sodium butyrate decreases the activation of NF- $\kappa \mathrm{B}$ reducing inflammation and oxidative damage in the kidney of rats subjected to contrast-induced nephropathy. Nephrol Dial Transplant. 2012;27(8):3136-3140. doi:10.1093/ndt/ gfr807

37. Kwasa EA, Vinayak S, Armstrong R. The role of inflammation in contrast-induced nephropathy. Br J Radiol. 2014;87(1041):20130738. doi:10.1259/bjr.20130738

38. Roffi M, Patrono C, Collet JP, et al. [2015 ESC guidelines for the management of acute coronary syndromes in patients presenting without persistent ST-segment elevation. Task force for the management of acute coronary syndromes in patients presenting without persistent ST-Segment Elevation of the European Society of Cardiology (ESC)]. G Ital Cardiol. 2016;17(10):831-872. Italian. doi:10.1714/2464.25804

39. Neumann FJ, Sousa-Uva M, Ahlsson A, et al. [2018 ESC/EACTS guidelines on myocardial revascularization. The task force on myocardial revascularization of the European Society of Cardiology (ESC) and European Association for Cardio-Thoracic Surgery (EACTS)]. G Ital Cardiol. 2019;20(7-8 Suppl 1):1S-61S. Italian. doi:10.1714/3203.31801

40. Arslan S, Yildiz A, Dalgic Y, et al. Avoiding the emergence of contrast-induced acute kidney injury in acute coronary syndrome: routine hydration treatment. Coron Artery Dis. 2021;32(5):397-402. doi:10.1097/MCA.0000000000000966

41. Nijssen EC, Nelemans PJ, Rennenberg RJ, et al. Impact on clinical practice of updated guidelines on iodinated contrast material: CINART. Eur Radiol. 2020;30(7):4005-4013. doi:10.1007/s00330-020-06719-7

42. Qian G, Fu Z, Guo J, et al. Prevention of contrast-induced nephropathy by central venous pressure-guided fluid administration in chronic kidney disease and congestive heart failure patients. JACC Cardiovasc Interv. 2016;9(1):89-96. doi:10.1016/j.jcin.2015.09.026

43. Maioli M, Toso A, Leoncini M, et al. Bioimpedance-guided hydration for the prevention of contrast-induced kidney injury: the HYDRA study. J Am Coll Cardiol. 2018;71(25):2880-2889. doi:10.1016/j.jacc.2018.04.022

44. Briguori C, D'Amore C, De Micco F, et al. Left ventricular end-diastolic pressure versus urine flow rate-guided hydration in preventing contrast-associated acute kidney injury. JACC Cardiovasc Interv. 2020;13(17):2065-2074. doi:10.1016/j.jcin.2020.04.051

45. Nijssen EC, Rennenberg RJ, Nelemans PJ, et al. Prophylactic hydration to protect renal function from intravascular iodinated contrast material in patients at high risk of contrast-induced nephropathy (AMACING): a prospective, randomised, phase 3, controlled, open-label, non-inferiority trial. Lancet. 2017;389(10076):1312-1322. doi:10.1016/S0140-6736(17)30057-0

46. Tepel M, van der Giet M, Schwarzfeld C, et al. Prevention of radiographic-contrast-agent-induced reductions in renal function by acetylcysteine. N Engl J Med. 2000;343(3):180-184. doi:10.1056/NEJM200007203430304

47. Merten GJ, Burgess WP, Gray LV, et al. Prevention of contrast-induced nephropathy with sodium bicarbonate: a randomized controlled trial. JAMA. 2004;291(19):2328-2334. doi:10.1001/jama.291.19.2328

48. Weisbord SD, Gallagher M, Jneid H, et al. Outcomes after angiography with sodium bicarbonate and acetylcysteine. $N$ Engl $J$ Med. 2018;378 (7):603-614. doi:10.1056/NEJMoa1710933

49. Trivedi H, Daram S, Szabo A, et al. High-dose N-acetylcysteine for the prevention of contrast-induced nephropathy. Am J Med. $2009 ; 122(9): 874$. e9-15. doi:10.1016/j.amjmed.2009.01.035

50. Xie W, Liang X, Lin Z, et al. Latest clinical evidence about effect of acetylcysteine on preventing contrast-induced nephropathy in patients undergoing angiography: a meta-analysis. Angiology. 2021;72(2):105-121. doi:10.1177/0003319720950162

51. Palli E, Makris D, Papanikolaou J, et al. The impact of N-acetylcysteine and ascorbic acid in contrast-induced nephropathy in critical care patients: an open-label randomized controlled study. Crit Care. 2017;21(1):269. doi:10.1186/s13054-017-1862-3

52. Iordache AM, Docea AO, Buga AM, et al. Sildenafil and tadalafil reduce the risk of contrast-induced nephropathy by modulating the oxidant/ antioxidant balance in a murine model. Food Chem Toxicol. 2020;135:111038. doi:10.1016/j.fct.2019.111038

53. Oh HJ, Oh H, Nam BY, et al. The protective effect of klotho against contrast-associated acute kidney injury via the antioxidative effect. Am J Physiol Renal Physiol. 2019;317(4):F881-F889. doi:10.1152/ajprenal.00297.2018

54. Yang KJ, Kim JH, Chang YK, et al. Inhibition of xanthine oxidoreductase protects against contrast-induced renal tubular injury by activating adenosine monophosphate-activated protein kinase. Free Radic Biol Med. 2019;145:209-220. doi:10.1016/j.freeradbiomed.2019.09.027

55. Walter DH, Dimmeler S, Zeiher AM. Effects of statins on endothelium and endothelial progenitor cell recruitment. Semin Vasc Med. 2004;4 (4):385-393. doi:10.1055/s-2004-869595

56. Leoncini M, Toso A, Maioli M, et al. Early high-dose rosuvastatin for contrast-induced nephropathy prevention in acute coronary syndrome: results from the PRATO-ACS study (Protective Effect of Rosuvastatin and Antiplatelet Therapy On contrast-induced acute kidney injury and myocardial damage in patients with Acute Coronary Syndrome). J Am Coll Cardiol. 2014;63(1):71-79. doi:10.1016/j.jacc.2013.04.105

57. Quintavalle C, Fiore D, De Micco F, et al. Impact of a high loading dose of atorvastatin on contrast-induced acute kidney injury. Circulation. 2012;126(25):3008-3016. doi:10.1161/CIRCULATIONAHA.112.103317

58. Toso A, Maioli M, Leoncini M, et al. Usefulness of atorvastatin ( $80 \mathrm{mg}$ ) in prevention of contrast-induced nephropathy in patients with chronic renal disease. Am J Cardiol. 2010;105(3):288-292. doi:10.1016/j.amjcard.2009.09.026

59. Park JH, Shin HJ, Choi JY, et al. Is there association between statin usage and contrast-associated acute kidney injury after intravenous administration of iodine-based contrast media in enhanced computed tomography? Eur Radiol. 2020;30(10):5261-5271. doi:10.1007/s00330020-06897-4

60. Zhang F, Lu Z, Wang F. Advances in the pathogenesis and prevention of contrast-induced nephropathy. Life Sci. 2020;259:118379. doi:10.1016/j. Ifs. 2020.118379

61. Boozari M, Hosseinzadeh H. Preventing Contrast-Induced Nephropathy (CIN) with herbal medicines: a review. Phytother Res. 2021;35 (3):1130-1146. doi:10.1002/ptr.6880

62. Cruz DN, Goh CY, Marenzi G, et al. Renal replacement therapies for prevention of radiocontrast-induced nephropathy: a systematic review. Am $J$ Med. 2012;125(1):66-78.e3. doi:10.1016/j.amjmed.2011.06.029

63. Lakhal K, Ehrmann S, Robert-Edan V. Iodinated contrast medium: is there a renal problem? A clinical vignette-based review. Crit Care. $2020 ; 24$ (1):641. doi:10.1186/s13054-020-03365-9 


\section{Publish your work in this journal}

The International Journal of General Medicine is an international, peer-reviewed open-access journal that focuses on general and internal medicine, pathogenesis, epidemiology, diagnosis, monitoring and treatment protocols. The journal is characterized by the rapid reporting of reviews, original research and clinical studies across all disease areas. The manuscript management system is completely online and includes a very quick and fair peer-review system, which is all easy to use. Visit http://www.dovepress.com/testimonials.php to read real quotes from published authors.

Submit your manuscript here: https://www.dovepress.com/international-journal-of-general-medicine-journal 